\title{
ASO Visual Abstract: Development and Validation of Web-Based Nomograms for Predicting Cause-Specific Mortality in Surgically Resected Nonmetastatic Invasive Breast Cancer: A Population- Based Study
}

\author{
Guangyong Chen, $\mathrm{MD}^{1}$, Mei Jia, $\mathrm{MD}^{1}$, Qingpeng Zeng, $\mathrm{MD}^{2}$, and Huiming $\mathrm{Zhang}, \mathrm{MD}^{3}$ \\ ${ }^{1}$ Department of Pathology, Beijing Friendship Hospital, Capital Medical University; National Clinical Research Center for \\ Digestive Diseases, Beijing, China; ${ }^{2}$ Department of Thoracic Surgery, National Cancer Center/National Clinical Research \\ Center for Cancer/Cancer Hospital, Chinese Academy of Medical Sciences and Peking Union Medical College, Beijing, \\ China; ${ }^{3}$ Department of General Surgery, Beijing Friendship Hospital, Capital Medical University; National Clinical \\ Research Center for Digestive Diseases, Beijing, China
}

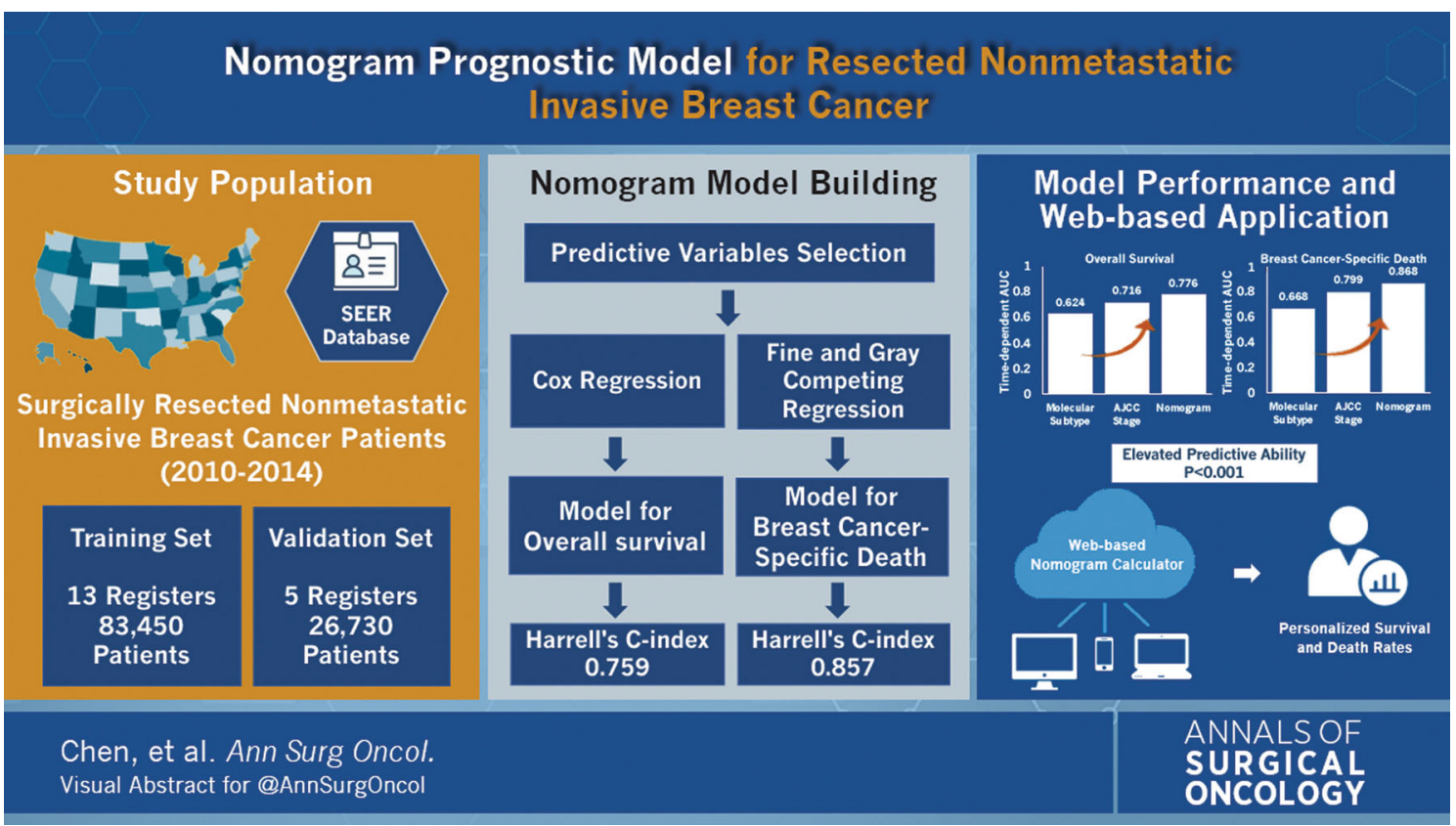

Our study reported on the construction of nomogram models to predict survival and cause-specific mortality in resected nonmetastatic invasive breast cancer based on the

Guangyong Chen and Mei Jia have contributed equally to this work.

(C) Society of Surgical Oncology 2021

Published Online: 2 June 2021

G. Chen, MD

e-mail: 13120193382@163.com population-based SEER database (https://doi.org/10.1245/ s10434-021-10129-4). These models perform well and could be accessed via a user-friendly website.

DISCLOSURE The authors have no conflicts of interest to declare.

Publisher's NoteSpringer Nature remains neutral with regard to jurisdictional claims in published maps and institutional affiliations. 